\title{
Avaliação Tardia por Tomografia Computadorizada Espiral de Enxertos de Pericárdio Bovino Liso como Substitutos Aórticos
}

\author{
Rena to A. K. Kalil, Carlos Jader Feldman, Fernando W. B. Ludwig, Alessandro D'A vila da Silva, \\ Paulo Roberto Prates, João R. M. Sant'Anna, Ivo A. N esralla \\ PortoAlegre, RS
}

Objetivo - Avaliar, morfologicamente, por tomografia computadorizada espiral, enxertos de pericárdio bovino liso empregados como substitutos aórticos.

Métodos - Dez pacientes foram submetidos a exame por tomografia computadorizada espiral para reconstituição da imagem dos enxertos. Os critérios de seleçãoforam tempo de seguimento superior a 2 anos, enxertos de pericárdio liso não revestido, implantados na aorta ascendente ou descendente.

Resultados - Os exames demonstraram bom resultado cirúrgico em todos os casos, persistindo em alguns, a imagem de dissecção aórtica distal à anastomose. Um caso apresentava hematoma entre o enxerto e a parede aórtica, em 5 não foram encontradas alterações estruturais no pericárdio e, nos demais, foi detectada dilatação de grau leve, em relação ao diâmetro descrito do enxerto implantado. Não foram vistos sinais de calcificação ou pseudoaneurismas.

Conclusão - Os enxertos tubulares de pericárdio bovino liso, não revestido, apresentam resultados satisfatórios quando empregados como substitutos aórticos. A médio prazo, não foram detectadas anormalidades estruturais relacionadas ao material empregado, pela tomografia computadorizada espiral, exceto dilatação em alguns casos.

Palavras-chave: tomografia computadorizada, aorta, enxertos, pericárdio bovino, heteroenxertos

\section{Late Evaluation of Bovine Pericardium Grafts as Aortic Substitutes by Spiral Com- puted Tomography}

Purpose - To evaluate morphologically by spiral computed tomography (SCT) bovine pericardial grafts as aortic substitutes.

Methods - Ten patients were submitted to aortic graft evaluation with SCT. There were 8 ascending and $2 \mathrm{de}$ scending grafts. Examination was done after 2 to 7 years post operative. Ages ranged from 49 to 67 years, with 6 male and 4 female patients. All had implanted smooth surface naked pericardial grafts.

Results - Surgical result was good in all. Distal dissection persisted in some cases. One had a peri-graft hematoma, 5 had no structural changes and 4 presented mild graft dilatation. There were no graft calcification or pseudoaneurysm.

Conclusion - Glutaraldehyde preserved naked smooth surface bovine pericardial aortic grafts present satisfactory structural aspect, as seen by SCT, at medium term follow-up. Mild dilatation may be seen in some cases.

Key-words: aortic grafts, bovine pericardium, computed tomography

Arq Bras Cardiol, volume 69 (nº 2), 111-115, 1997

Os primeiros relatos de interposição de enxertos aórticos foram descritos no final da década de 40 e início da

Instituto de Cardiologia do Rio Grande do Sul/Fundação Universitária de Cardiologia - Porto Alegre

Correspondência: Renato A. K. Kalil - Unidade de Pesquisa - Av. Princesa Isabel, 395 - 90620-001 - Porto Alegre, RS

Recebido para publicação em 17/3/97

Aceito em 19/6/97 década de 50, utilizando-se enxertos arteriais humanos (homólogos) preservados ${ }^{1-3}$. As próteses sintéticas foram introduzidas a partir de $1952^{4,5}$. Atualmente, têm sido empregadas, nas cirurgias de substituição de aorta ascendente e torácica descendente, enxertos tubulares de dácron, cuja evolução tardia é conhecida e bem aceita, sendo relatadas, em média, sobrevidas de $70 \%$, em 5 anos, e de $60 \%$, em 10 $\operatorname{anos}^{6-9}$. 
Mais recentemente, foram desenvolvidos enxertos de pericárdio bovino preservados em glutaraldeído, a partir da tecnologia empregada na produção de biopróteses valvulares, enxertos que são implantados em alguns centros com resultados clínicos muito bons, a curto e médio prazos ${ }^{10-14}$, comparáveis aos obtidos com o emprego de enxertos de dácron. Questiona-se, entretanto, a sua durabilidade a médio e longo prazos, bem como possíveis degenerações, calcificações, formação de pseudoaneurismas, dilatação e rupturas do enxerto.

O método da tomografia computadorizada espiral gera, a partir de aproximadamente 30 s de exposição, excelentes imagens de reconstituição de estruturas vasculares, em três dimensões e a cores, e detecta com muita sensibilidade as calcificações. Assim, pode-se analisar a estrutura morfológica dos enxertos implantados.

Caso demonstrem boa durabilidade, os enxertos de pericárdio bovino seriam preferíveis aos de dácron por serem impermeáveis, macios e flexíveis, o que facilitaria a feitura das anastomoses, resultando, em linha de sutura, mais hemostática e reduzindo os problemas de sangramento ${ }^{15}$. Além do simples manuseio cirúrgico e técnica mais vantajosa, os enxertos de pericárdio são de baixo custo de produção, o que reduziria o ônus das despesas hospitalares dos sistemas de saúde.

Este trabalho tem por objetivo avaliar, morfologicamente, por tomografia computadorizada espiral, os enxertos tubulares de pericárdio bovino liso empregados como substituto aórtico em aneurismas e dissecções da aorta.

\section{Métodos}

Para avaliação dos enxertos tubulares de pericárdio bovino liso foram selecionados, entre aqueles com mais de dois anos de acompanhamento pós-operatório (PO), 10 pacientes, que foram submetidos a exame por tomografia computadorizada espiral para reconstituição da imagem dos enxertos. O equipamento utilizado foi um Spiral CT, marca Elscint, mod. Twin.

Administrou-se contraste endovenoso em veia periférica do membro superior, através de um injetor automático, tendo sido ajustado um fluxo de $2 \mathrm{~mL} / \mathrm{s}$. O total de contraste para cada paciente foi de $80 \mathrm{~mL}$. Obteve-se um scout inicial e foram programados cortes desde acima do arco aórtico, até o diafragma com intervalos de $6 \mathrm{~mm}$. Com um único movimento respiratório e retardo de 15 s obtiveram-se imagens de todo o mediastino. O tempo médio de permanência do paciente na mesa de exames foi de 32 s. Em seguida, foram reconstruídas imagens em três dimensões, em todos os planos.

O pericárdio bovino utilizado na confecção das biopróteses é coletado em frigoríficos logo após o abate dos animais e colocado em solução hipertônica de $\mathrm{NaCl}$ a pH 7,4 $\mathrm{a} 4^{\circ} \mathrm{C}$. No laboratório, é submetido à fixação em solução de glutaraldeído a $0,5 \%$ por 15 dias, a $4^{\circ} \mathrm{Ce}$, durante três dias, à temperatura ambiente, com trocas periódicas da solução. Concluída esta fase, o pericárdio bovino é colocado em solução de formaldeído a 4\% para conservação, sendo realiza- dos os testes de controle de qualidade, entre eles, encolhimento, alongamento, rotura, tensão superficial crítica, medida da espessura e histologia. São liberados apenas os pericárdios que preencherem os critérios previamente estabelecidos.

Assim processado, o pericárdio é empregado na confecção de válvulas e tubos. Para a confecção da válvula são utilizados suportes semi-rígidos ${ }^{10}$. Os tubos são apresentados lisos ou corrugados ${ }^{11}$, conforme a membrana mantenha-se na forma natural ou tenha sido submetida a pregueamento à semelhança dos tubos de dácron.

Os critérios para seleção dos pacientes desta série foram: tempo de seguimento superior a dois anos pós-implante de enxerto de pericárdio bovino liso, não revestido, implantado na aorta ascendente ou torácica descendente.

Dos 10 pacientes analisados, sete apresentavam dissecção aórtica tipo A, um dissecção aórtica tipo B, um aneurisma de aorta ascendente e um aneurisma de aorta descendente. Insuficiência valvular aórtica esteve presente em dois pacientes com dissecção aórtica tipo A. A idade variou de 49 a 66 (58 \pm 6$)$ anos, sendo seis do sexo masculino e quatro do feminino. Tempo de seguimento foi de dois a sete anos PO.

Nos casos de dissecção aórtica tipo A e aneurisma de aorta ascendente, a abordagem cirúrgica foi através de esternotomia mediana, utilizando-se circulação extracorpórea, hipotermia sistêmica e cardioplegia. A abordagem no caso de dissecção aórtica tipo B foi por toracotomia lateral esquerda.

Condutos avalvulados foram utilizados para substituição da aorta ascendente quando a válvula aórtica foi preservada. Em seis pacientes empregou-se enxerto tubular de pericárdio bovino liso $27 \mathrm{~mm}$, sendo em dois, valvulados. Dos quatro enxertos restantes, dois eram tubos de pericárdio $25 \mathrm{~mm}$, um com diâmetro de $31 \mathrm{~mm}$ e no paciente com dissecção aórtica tipo B utilizou-se retalho de pericárdio bovino logo distal à emergência da artéria subclávia esquerda.

As anastomoses foram realizadas com fio de polipropileno 3-0 e foi feito reforço das bordas da incisão com feltro de teflon. As linhas de sutura foram cuidadosamente inspecionadas e utilizados pontos adicionais quando necessários para hemostasia.

As imagens foram analisadas em conjunto pelo cirurgião e o radiologista, em busca de possíveis indícios de calcificações, dilatações ou outras alterações estruturais nos enxertos implantados.

\section{Resultados}

As imagens demonstraram bom resultado cirúrgico nos 10 casos estudados. Em um paciente havia formação de hematoma organizado entre as paredes do enxerto e da aorta que o recobria, na face lateral direita, sem comprometer o diâmetro do lúmen. Em alguns pacientes persistia a imagem de dissecção aórtica distal à anastomose, porém sem progresso da dilatação ou formação de aneurismas. Um enxerto, fixado distalmente pela técnica sem sutura, mostrava 
sensível estreitamento nesse local, porém sem estenose com significação hemodinâmica (fig. 1). Foram detectadas algumas calcificações da parede aórtica em áreas não relacionadas aos enxertos, como no arco aórtico e aorta torácica descendente. Em cinco pacientes o diâmetro do tubo era semelhante ao descrito no ato cirúrgico. Em um caso não era enxerto tubular, não sendo encontradas alterações. Nos demais quatro, houve leve dilatação (fig. 2), onde a média no implante foi $27,5 \pm 0,8 \mathrm{mme}$ a média na avaliação $35,2 \pm 3,6 \mathrm{~mm}$ de diâmetro do tubo examinado. Os resultados estão sumarizados na tabela I.

\section{Discussão}

Enxertos de dácron constituem os substitutos aórticos mais utilizados em qualquer situação. Seu desempe-

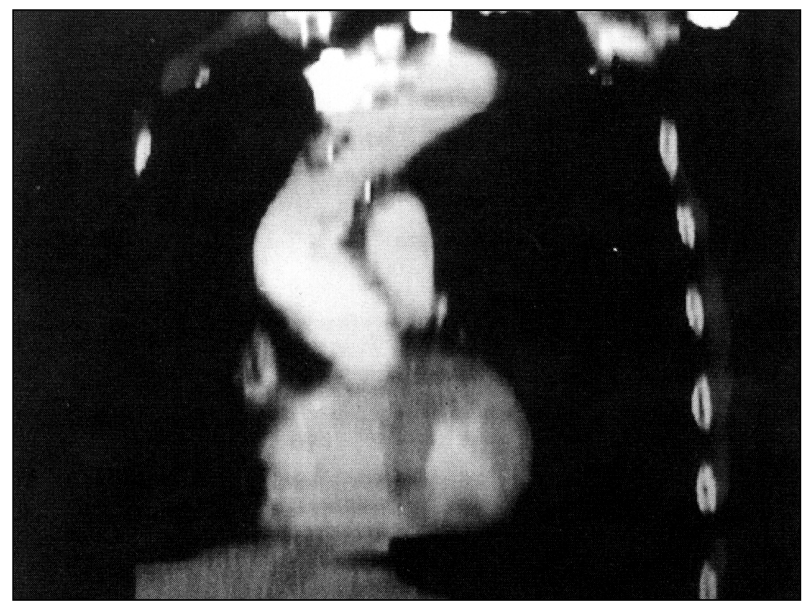

Fig. 1 - Reconstrução bidimensional da aorta ascendente, observa-se a mesma substituída por tubo de pericárdio bovino valvulado sem alterações notáveis. Há estreitamento na anastomose distal devido à fixação pela técnica sem sutura.

\begin{tabular}{|c|c|c|c|c|c|c|}
\hline Caso & Idade/sexo & Diagnóstico & $\begin{array}{l}\text { Data da } \\
\text { cirurgia }\end{array}$ & $\begin{array}{l}\text { Tempo } \\
\text { PO } \\
\text { (anos) }\end{array}$ & Aspecto do enxerto & Observações \\
\hline 1 & $52 \mathrm{M}$ & $\begin{array}{l}\text { Aneurisma e dissecção } \\
\text { aguda de aorta ascen- } \\
\text { dente com insuficiência } \\
\text { valvular. Tipo A. }\end{array}$ & $22 / 5 / 90$ & 6 & $\begin{array}{l}\text {-Sem alterações; } \\
\text {-Sem calcificações. }\end{array}$ & $\begin{array}{l}\text {-Enxerto tubular de PB bovino } \\
\text { liso } 27 \mathrm{~mm} \text { com válvula. } \\
\text {-Dissecção nos óstios coronários. }\end{array}$ \\
\hline 2 & $57 \mathrm{M}$ & $\begin{array}{l}\text { Dissecção aguda de aorta } \\
\text { ascendente. Tipo A. }\end{array}$ & $25 / 8 / 92$ & 4 & - Dilatação do tubo: $3,2 \times 3,7 \mathrm{~cm}$. & $\begin{array}{l}\text { Enxerto tubular de PB liso } 27 \mathrm{~mm} \\
\text { sem válvula. }\end{array}$ \\
\hline 3 & $66 \mathrm{M}$ & $\begin{array}{l}\text { Aneurisma verdadeiro de } \\
\text { aorta ascendente. }\end{array}$ & $18 / 9 / 92$ & 4 & $\begin{array}{l}\text { - Hematoma extra-tubo; } \\
\text { - Sem calcificações; } \\
\text { - Dilatação do tubo: } \\
\text { 2,7x2,0mm. }\end{array}$ & $\begin{array}{l}\text { - Enxerto tubular de PB liso } \\
25 \mathrm{~mm} \text { sem válvula. } \\
\text {-Massa cística junto à aorta em } \\
\text { sua parede lateral direita. }\end{array}$ \\
\hline 4 & $67 \mathrm{~F}$ & $\begin{array}{l}\text { Dissecção aguda de aorta } \\
\text { ascendente. Tipo A. }\end{array}$ & $21 / 3 / 93$ & 3 & $\begin{array}{l}\text { Persiste dissecção de aorta } \\
\text { ascendente. }\end{array}$ & $\begin{array}{l}\text {-Enxerto tubular de PB liso } \\
31 \mathrm{~mm} \text { sem válvula. }\end{array}$ \\
\hline 5 & $64 \mathrm{~F}$ & $\begin{array}{l}\text { Dissecção aguda de } \\
\text { aorta ascendente. }\end{array}$ & $01 / 8 / 90$ & 6 & $\begin{array}{l}\text { - Sem alterações } \\
\text { - Sem calcificações }\end{array}$ & $\begin{array}{l}\text { - Enxerto tubular de PB liso } \\
\text { sem valvula }\end{array}$ \\
\hline 6 & $55 \mathrm{M}$ & $\begin{array}{l}\text { Aneurisma sacular roto } \\
\text { de aorta descendente }\end{array}$ & $10 / 1 / 89$ & 7 & $\begin{array}{l}\text { - Dilatação na aorta } \\
\text { descendente } \\
\text { - Calcificação na aorta } \\
\text { descendente }\end{array}$ & $\begin{array}{l}\text { - Enxerto retalho de PB liso logo } \\
\text { abaixo da subclávia esquerda. }\end{array}$ \\
\hline 7 & $50 \mathrm{~F}$ & $\begin{array}{l}\text { Dissecção aguda de } \\
\text { aorta ascendente } \\
\text { Tipo A }\end{array}$ & $21 / 2 / 93$ & 3 & $\begin{array}{l}\text { - Dilatação do tubo: } 3,0 \mathrm{~cm} \\
\text { - Presença gânglios } \\
\text { mediastinais }\end{array}$ & $\begin{array}{l}\text { - Enxerto tubular de PB liso } 27 \mathrm{~mm} \\
\text { sem válvula }\end{array}$ \\
\hline 8 & $59 \mathrm{~F}$ & $\begin{array}{l}\text { Dissecção aguda de } \\
\text { aorta ascendente } \\
\text { Tipo A }\end{array}$ & $11 / 12 / 92$ & 4 & - Calcificação do arco aórtico; & $\begin{array}{l}\text { - Enxerto tubular de PB liso } \\
27 \mathrm{~mm} .\end{array}$ \\
\hline 9 & $49 \mathrm{M}$ & $\begin{array}{l}\text { Dissecção aórtica } \\
\text { Tipo A }\end{array}$ & $23 / 11 / 89$ & 7 & $\begin{array}{l}\text { - Calcificação distal } \\
\text { - Calcificação de aorta } \\
\text { descendente e traquéia; } \\
\text { - Dilatação do tubo: } 4,0 \mathrm{~cm} \text {. }\end{array}$ & - Enxerto tubo de PB liso \\
\hline 10 & $51 \mathrm{M}$ & $\begin{array}{l}\text { Dissecção aguda de } \\
\text { aorta descendente } \\
\text { Tipo B. }\end{array}$ & $11 / 08 / 91$ & 5 & Sem alterações & - Enxerto retalho de PB. \\
\hline
\end{tabular}




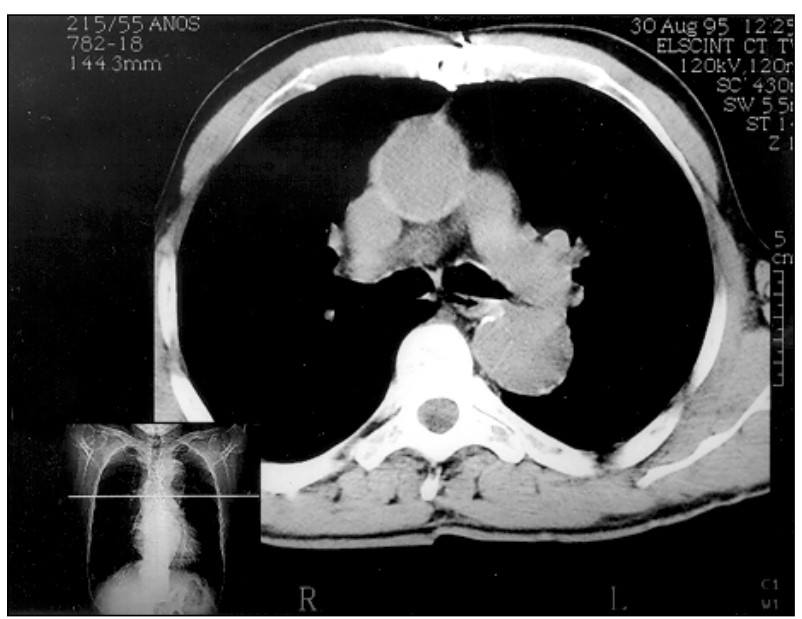

Fig. 2 - Corte tomográfico transversal na altura da porção média da aorta ascendente, observando-se o enxerto tubular com dilatação leve, sem calcificações. A aorta descendente é dilatada, apresentando parede calcificada.

nho a longo prazo é estabelecido e bem conhecido. Inicialmente, sua porosidade causava problemas de hemostasia, porém, mais recentemente, enxertos de baixa porosidade minimizaram esta questão. A maleabilidade também melhorou em enxertos de novas gerações. O custo, entretanto, permanece elevado, onerando significativamente os sistemas de saúde e, muitas vezes, impedindo seu emprego. Sabe-se que os enxertos de dácron podem apresentar alguma dilatação na evolução tardia, sem significado clínico.

O pericárdio bovino preservado em glutaraldeído é utilizado há duas décadas na fabricação de biopróteses valvulares. Seu desempenho, nesses implantes, também é conhecido. Há calcificações precoces em pacientes jovens, mas em faixas etárias acima dos 40 anos, a incidência de degeneração tecidual é pequena, sendo um substituto valvular universalmente aceito. Por analogia, passou-se a produzir enxertos tubulares de pericárdio bovino submetido ao mesmo método de preparação das biopróteses. Suas vantagens seriam a impermeabilidade, a maleabilidade e o baixo custo.

Há cerca de 10 anos, utilizam-se, largamente em nosso país, enxertos tubulares de pericárdio bovino, como substituto aórtico. Os resultados imediatos e a médio prazo têm sido muito bons. $\mathrm{O}$ desempenho tardio, devido à possibilidade de degeneração, como ocorre com as biopróteses é preocupante. A faixa etária de ocorrência de aneurismas e dissecções seria um fator a minimizar o problema da falha tecidual do pericárdio, pois sabe-se que acima dos 40 anos de idade, a incidência de alterações primárias do tecido biológico implantadoé menor.

Há relatos escassos e informais de reoperações por ruptura de enxertos, porém nos faltam estudos clínicos evolutivos deste problema.

Nesta série, procuramos avaliar pacientes cuja evolução foi favorável, por um método que poderia revelar alterações estruturais degenerativas dos enxertos. Além de dilatação leve, fato que ocorre igualmente com enxertos de dácron, não encontramos sinais de degeneração ou calcificação do pericárdio bovino em pacientes com até seis anos de evolução. Este fato nos dá uma perspectiva animadora, embora com cautela necessária, de que este substituto aórtico possa apresentar bom desempenho tardio. $\mathrm{Na}$ maioria das vezes, utiliza-se a técnica da inclusão, ou seja, aquela na qual a parede do aneurisma é utilizada para recobrir o enxerto implantado, contribuindo como apoio para reduzir o processo degenerativo e, eventualmente, suportar o enxerto, mesmo no caso de haver degeneração. Em outros casos, os enxertos são fornecidos com uma tela externa de dácron que serve de suporte, sem elevar o custo, mantendo a impermeabilidade. A preocupação com a possível degeneração tardia pode assim ser reduzida pela observância desses detalhes técnicos.

De qualquer maneira, os pacientes devem ser acompanhados clinica e radiologicamente, não podendo ser considerados "curados" do problema pela cirurgia. Tanto mais que a doença pode evoluir também em outras porções da aorta.

Em conclusão, os enxertos aórticos de pericárdio bovino preservado em glutaraldeído, mostraram boa estrutura morfológica, à luz da tomografia computadorizada espiral, sem sinais degenerativos, justificando-se a manutenção de seu emprego como substituto aórtico, com as cautelas técnicas e clínicas pertinentes a um tecido cuja evolução mais tardia neste uso ainda não é conhecida. O significado da dilatação do enxerto tubular, observada em alguns casos, não está claro e merece avaliação futura.

\section{Agradecimentos}

À Unidade de Pesquisa do Instituto de Cardiologia do Rio Grande do Sul/Fundação Universitária de Cardiologia, aos Drs. Guraracy F. Teixeira F ${ }^{\circ}$, Rogério Abrahão, Orlando C. Wender, Flávio P. Oliveira, Edemar M. C. Pereira, MarisaF. Santos, Raul F. A. Lara e Altamiro Reis da Costa pela colaboração prestada.

\section{Referências}

1. DubostC, Allary M, Deconomos N-Ressection of an aneurysm of the abdominal aorta: reestablishment of the continuity by a preserved human arterial graft with result after five months. Arch Surg 1952; 64: 405-8.

2. Gross RE, Bill Jr AH, Pierce EC - Methods for preservation and transplantation of arterial grafts: observations on arterial grafts in dogs. Report of transplantation of preserved arterial grafts in nine human cases. Surg Gynecol Obstet 1949; 88: 689-701
3. Oudot J - La greffe vasculaire dans les thromboses du carrefour aortic. Press Med 1951; 59: 234-6.

4. Lindenauer SM - The fabric vascular prosthesis. In: Rutherford RB. ed - Vascular Surgery. $3^{\text {trd }}$ ed. Philadelphia: WB Saunders, 1989; 1: 450.

5. Lindenauer SM, Weber TR, Miller TA et al - The use of reelour as a vascular prosthesis. Biomed Eng 1976; 11: 301-6.

6. Culliford AT, Ayvaliotis B, Shemin R, Colvin SB, Isom OW, Spencer FC - Aneu- 
rysms of the ascending aorta and transverse arch: surgical experience in $80 \mathrm{pa}-$ tients. J Thoracic Cardiovasc Surg 1982; 83: 701-10.

7. Fontes RD, Stolf NAG, Lourenço DD et al - Dez anos de cirurgias dos aneurismas e dissecções crônicas de aorta ascendente no Instituto do Coração FMUSP. Rev Bras Cir Cardiovasc 1991; 6: 24-9.

8. Ecloff L, Rothlin M, Kugelmeier J, Senning A, Turina M - The ascending aortic aneurysm: replace or repair? Ann Thorac Surg 1982; 34: 117-24.

9. Raudkivi PJ, Williams JO, Monro JL, Ross JK - Surgical treatment of the ascending aorta: fourteen years' experience with 83 patients. J Thorac Cardiovasc 1989; 675-82.

10. Santos JV, Braile DM, Soares MJF - Avaliação de tubo valvado de pericárdio bovino em um modelo experimental animal. Rev Bras Cir Cardiovasc 1990; 5: 1625 .

11. Salles CA, Puig LB, Casagrande IS et al - Early experience with crimped bovine pericardial conduit for arterial reconstruction. Eur J Cardio-Thoracic Surgery
1991; 5: 273-9.

12. Ardito RV, Santos JLV, Mayorquim RC - Substituição completa da aorta ascendente e da valva aórtica com tubo valvado de pericárdio bovino. Rev Bras Cir Cardiovasc 1987; 2: 129-38.

13. Gonzalez MZ, Kalil RAK, Prates PR - Resultados a médio prazo da correção de aneurismas da aorta ascendente com enxertos de pericárdio bovino. Anais do III Congresso Gaúcho de Cirurgia Cardíaca. Porto Alegre: Sociedade Brasileira de Cirurgia Cardiovascular, 1994.

14. Fantini FA, Bayard GF, Vrandecic M - Substituição da aorta ascendente e arco aórtico por condutos de pericárdio bovino: resultados a médio prazo. Anais do XXI Congresso Nacional de Cirurgia Cardíaca. Porto Alegre: Sociedade Brasileira de Cirurgia Cardiovascular, 1994.

15. Ardito RV, Santos JLV, Mayorquim RC et al - Substituição completa da aorta ascendente e da valva aórtica com tubo valvulado de pericárdio bovino. Rev Bras Cir Cardiovasc 1987; 12: 129-38. 